\title{
Growth hormone retesting during puberty: a cohort study
}

\author{
Paolo Cavarzere', Rossella Gaudino ${ }^{1,2}$, Marco Sandri ${ }^{3}$, Diego Alberto Ramaroli', Angelo Pietrobelli,2, \\ Marco Zaffanello ${ }^{1,2}$, Alessandra Guzzo ${ }^{4}$, Gian Luca Salvagno4, Giorgio Piacentini ${ }^{1,2}$ and Franco Antoniazzi ${ }^{1,2,5}$
}

${ }^{1}$ Pediatric Division, Department of Pediatrics, University Hospital of Verona, Verona, Italy, ${ }^{2}$ Pediatric Clinic, Department Surgical Sciences, Dentistry, Gynecology and Pediatrics, University of Verona, Verona, Italy, ${ }^{3}$ Data Methods and Systems Statistical Laboratory, University of Brescia, Brescia, Italy, ${ }^{4}$ Laboratory Unit, Department of Neurosciences, Biomedicine and Movement Sciences, University of Verona, Verona, Italy, and ${ }^{5}$ Regional Center for the Diagnosis and Treatment of Children and Adolescents Rare Skeletal Disorders, Pediatric Clinic, Department of Surgical Sciences, Dentistry, Gynecology and Pediatrics, University of Verona, Verona, Italy

Correspondence should be addressed to P Cavarzere Email paolocavarzere@yahoo.it

\begin{abstract}
Objectives: To report the frequency and characteristics of growth hormone (GH) deficiency (GHD) in adolescents who had normalized GH secretion at mid-puberty and to identify possible factors predictive for GH sufficiency at puberty. Design: Clinical analysis of children affected by GHD at five time points: diagnosis; first year of therapy; intermediate stage of puberty; retesting and end of growth phase.

Methods: The study population was 80 children with idiopathic GHD and treated with GH for at least 2 years. Treatment was discontinued at the intermediate stage of puberty. Retesting with an arginine test was performed 12 weeks later. If $\mathrm{GH}$ peak at retesting was $\geq 8 \mu \mathrm{g} / \mathrm{L}$, the therapy was definitively discontinued, otherwise it was restarted and continued until achievement of near-final height.

Results: GH therapy was discontinued in 44 children (55\%), and restarted in 36 (45\%). No evidence of differences in definitive height and in the delta height between the genetic target and the definitive height was found between the two groups. The only predictive factor for GHD at mid-puberty was the insulin growth factor-1 (IGF-1) level at 1 year of $\mathrm{GH}$ treatment.

Conclusions: GH secretion should be retested at mid-puberty. Retesting at puberty may reduce potential side effects and minimize costs, without impairing growth potential and final height.
\end{abstract}

\section{Introduction}

Growth hormone (GH) deficiency (GHD) is the most common pituitary hormone deficiency and the most frequent endocrine disorder manifesting with short stature in childhood (1). Its frequency is around 1:35001:4000 $(2,3)$. Diagnosis is based on absent or low levels of $\mathrm{GH}$ in response to stimulation with provocative tests (4). In Italy, GHD is diagnosed when peak GH levels are $<8 \mu \mathrm{g} / \mathrm{L}$ on two provocative tests, which concur with poor linear growth and delayed skeletal age (1). Children with GHD receive GH therapy at least until the end of growth (bone age of 14 years for females and 16 for males) and (c) 2020 European Society of Endocrinology Printed in Great Britain are then retested. The majority of children diagnosed with GHD display normal secretion when pharmacological tests are repeated at the end of growth and puberty $(5,6)$.

During GH treatment, catch-up growth is achieved during the first year of therapy and particularly during childhood (before 10 years of age); conversely, no additional gain in near-adult height seems to be achieved during puberty $(7,8)$. This is consistent with the idea that sensitivity to GH is greater during childhood and that therapeutic efforts to maximize height should be concentrated in the prepubertal years $(9,10,11)$. Patients

Published by Bioscientifica Ltd. 
with an uncomplicated course, whose height was normal or tall when spontaneous puberty occurred or was induced, achieve taller near-adult height $(8,12)$. Endogenous GH secretion increases physiologically during puberty and a correlation between GH levels and pubertal stages can be stated (13). If the hypothesis for a transient prepubertal GH deficiency is true, it would make sense to retest GH secretion at puberty, when its secretion rises rather than at the end of the growth period $(6,14)$. In this way, unnecessary treatment during puberty in children with a normalized GH secretion could be avoided, potential side effects of the therapy (e.g. idiopathic intracranial hypertension, slipped capital femoral epiphysis, scoliosis, pancreatitis, transient gynecomastia, disorders of glucose tolerance) minimized and costs of treatment reduced (15).

With this study, we want to report the frequency and characteristics of GHD adolescents who had normalized GH secretion at puberty. The secondary aim is to identify possible predictive factors for GH sufficiency at puberty.

\section{Patients and methods}

\section{Patients}

In this retrospective study, we have enrolled 80 prepubertal patients (46 males and 34 females) with idiopathic GHD referred to the Pediatric Endocrinology Division of the Hospital of Verona, Italy, for GH treatment between 2004 and 2014. At diagnosis, all patients underwent two different provocative tests with arginine, insulin and/ or glucagon, both of them showing a GH peak of $<8$ $\mu \mathrm{g} / \mathrm{L}$. Brain MRI resulted normal in all patients. There was no evidence of other hypothalamic-pituitary axis deficiencies. GH treatment with $0.033 \mathrm{mg} / \mathrm{kg} / \mathrm{day}, 6$ days per week for at least two years, was begun immediately after diagnosis. The GH dose was progressively adjusted to the weight increase, so the dose $\mathrm{kg}$ /day remained constant for all patients at least during the first year of treatment.

Treatment was discontinued when patients reached the intermediate stage of puberty according to Tanner stage 3 (testicular volume $\geq 10 \mathrm{~mL}$ for males, B3 for females). After 12 weeks of treatment withdrawal, GH secretion was retested using a provocative test with arginine. If the peak GH was $\geq 8 \mu \mathrm{g} / \mathrm{L}$, therapy was definitively discontinued, otherwise it was restarted. Figure 1 presents the inclusion and exclusion criteria and a study flowchart.

Assessment with clinical, auxological and biochemical parameters was performed at five time points: at diagnosis; after the first year of therapy; at the intermediate stage of puberty; at retesting and at the end of the follow-up when near-adult height was achieved.

Evaluation included measurement of body weight, height, body-mass index (BMI, weight in kg divided by height in meters squared) and pubertal development according to the Tanner and Whitehouse method (16). For boys, testicular volume was measured using Prader's orchidometer. Height was measured with a Harpenden stadiometer and expressed in centimeters. Familiar genetic target was calculated based on the parents' height. At diagnosis and every year thereafter, all children underwent serum IGF-1 measurement and radiography to determine bone age (BA), evaluated by the same pediatric endocrinologist using the Greulich and Pyle method (17).

The study was conducted in compliance with the terms of the Helsinki II Declaration. The Institutional Ethics Committee of the provinces of Verona and Rovigo, Italy, took note of the retrospective conduct of the study and approved it in order to publish the results. Written informed consent was obtained from the parents of each patient.

\section{Assay}

Serum IGF-1 was measured using a one-step sandwich chemiluminescence immunoassay (CLIA, LIAISON Analyzer, Diasorin, Vercelli, Italy). Analytical sensitivity was $3 \mathrm{ng} / \mathrm{mL}$. Intra-assay and inter-assay coefficients of variation were $4.3 \%$ and $7.1 \%$, respectively. Serum GH was measured using a sandwich chemiluminescence immunoassay (CLIA, LIAISON Analyzer, Diasorin, Vercelli, Italy). Analytical sensitivity was $<0.01 \mathrm{ng} / \mathrm{mL}$. Intra-assay and inter-assay coefficients of variation were $4.4 \%$ and $6.6 \%$, respectively.

At diagnosis, GH was evaluated after provocative testing with arginine, insulin or glucagon; at retesting a single arginine, provocative test was used. The tests were performed between $0800 \mathrm{~h}$ and $0900 \mathrm{~h}$ after overnight fasting, on separate days (at least 2 days apart).

Arginine was administered intravenously $(0.5 \mathrm{~g} /$ $\mathrm{kg}$, max $30 \mathrm{~g}$ ) over $30 \mathrm{~min}$ and blood samples for $\mathrm{GH}$ determination were collected at 0, 30, 60, 90 and $120 \mathrm{~min}$. For the insulin tolerance test, insulin was administered intravenously $(0.08 \mathrm{U} / \mathrm{kg}, \max 2 \mathrm{U})$ at time zero, and blood samples for $\mathrm{GH}$ and glucose determination were collected at $0,10,15,20,30$ and $60 \mathrm{~min}$. Glucagon was administered intramuscularly $(0.1 \mathrm{mg} / \mathrm{kg}$, max. $1 \mathrm{mg})$. Blood samples for $\mathrm{GH}$ and glucose were collected at baseline and then at 30,60, 90, 120 and 180 min after glucagon administration. 


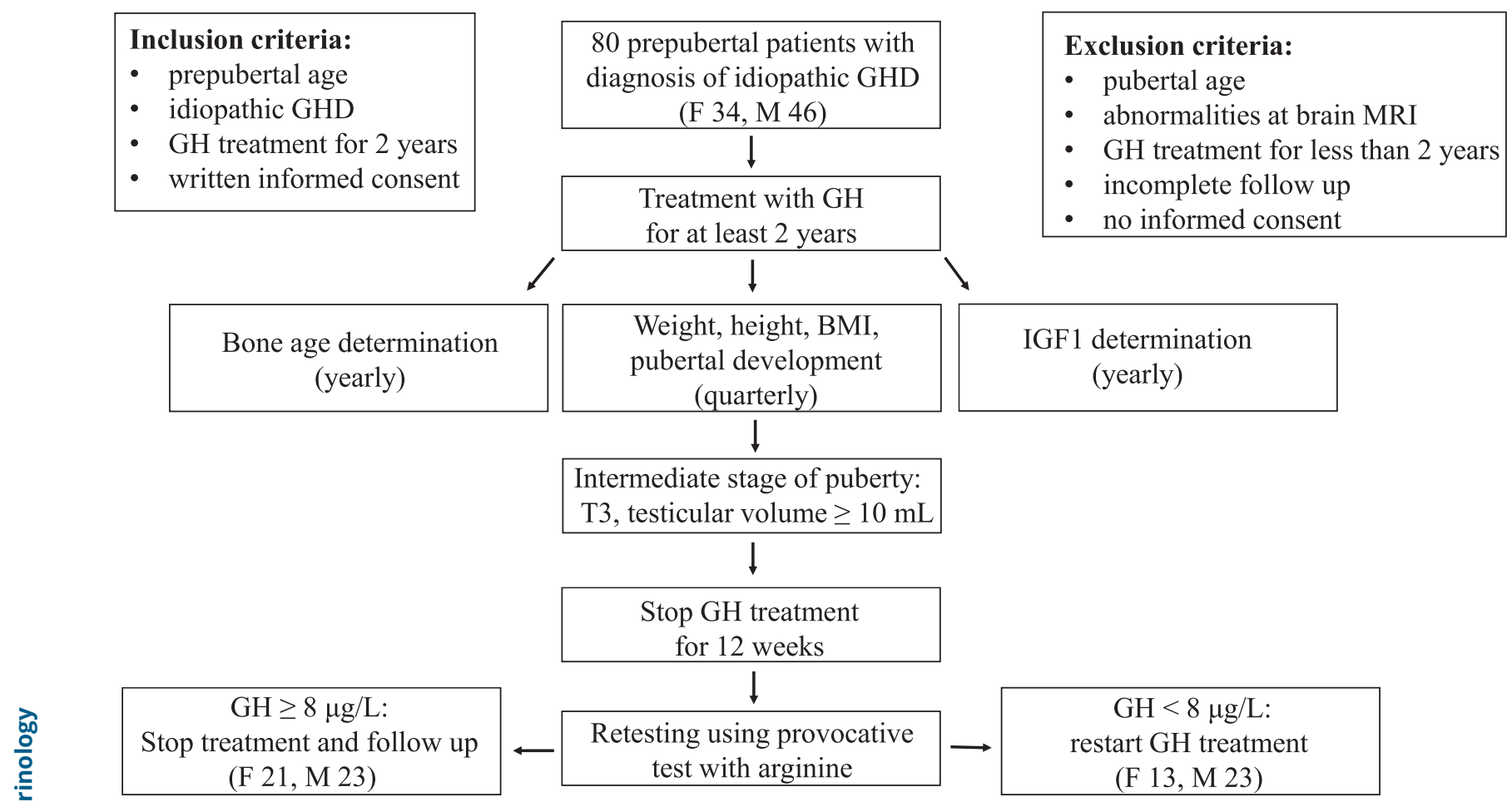

Figure 1

Study flowchart showing inclusion and exclusion criteria.

Priming with sex steroids prior to GH stimulation test was not used. All children underwent brain MRI after the diagnosis of GHD.

\section{Statistical analysis}

We performed descriptive and comparative analysis of clinical and auxological characteristics for males and females. Categorical variables were summarized as frequency and percentage. Numerical variables were summarized by median and interquartile range and were compared across independent groups by the Wilcoxon rank-sum test. The associations between IGF-1 levels (at diagnosis, after 1 year of treatment) and clinical/auxological characteristics were evaluated using Spearman's rank correlation and modeled by linear regression (Fig. 2).

The relationships between the risk of persistent GHD at mid-puberty and clinical/auxological characteristics, evaluated at the preceding timepoints, were investigated using logistic regression. The relationship between IGF-1 (and IGF-1 SDS) and the risk of GHD was modeled using logistic regression with a linear spline (with one knot), that is, using a piecewise linear relationship with two straight lines joined at a breakpoint. The predictive performance of this model was assessed by estimating the area under the ROCs (AUC); the optimal cutoffs for IGF-1 SDS were identified by maximizing the Youden Index. Sensitivity and specificity for these thresholds were reported.

Statistical analysis was performed using Stata15 (StataCorp 2017) and R (version 3.6.1; R Foundation for Statistical Computing, Vienna, Austria) software. A two-tailed test with $P<0.05$ was considered statistically significant.

\section{Results}

Table 1 presents the clinical data at diagnosis stratified by gender (stratification by study group is given in Supplementary Table 1 , see section on supplementary materials given at the end of this article). Age and auxological data are similar for the males and the females. Immediately after the diagnosis of GHD, treatment with $\mathrm{GH}$ was begun at a dose of $0.033 \mathrm{mg} / \mathrm{kg}$; no side effects were reported. Therapy was definitively discontinued after retesting $\mathrm{GH}$ secretion at mid-puberty in 44 children (55\%, group A), whereas $\mathrm{GH}$ treatment was restarted in 36 children (45\%, group B) (Fig. 1). Table 2 summarizes the patients' data during treatment stratified by gender 


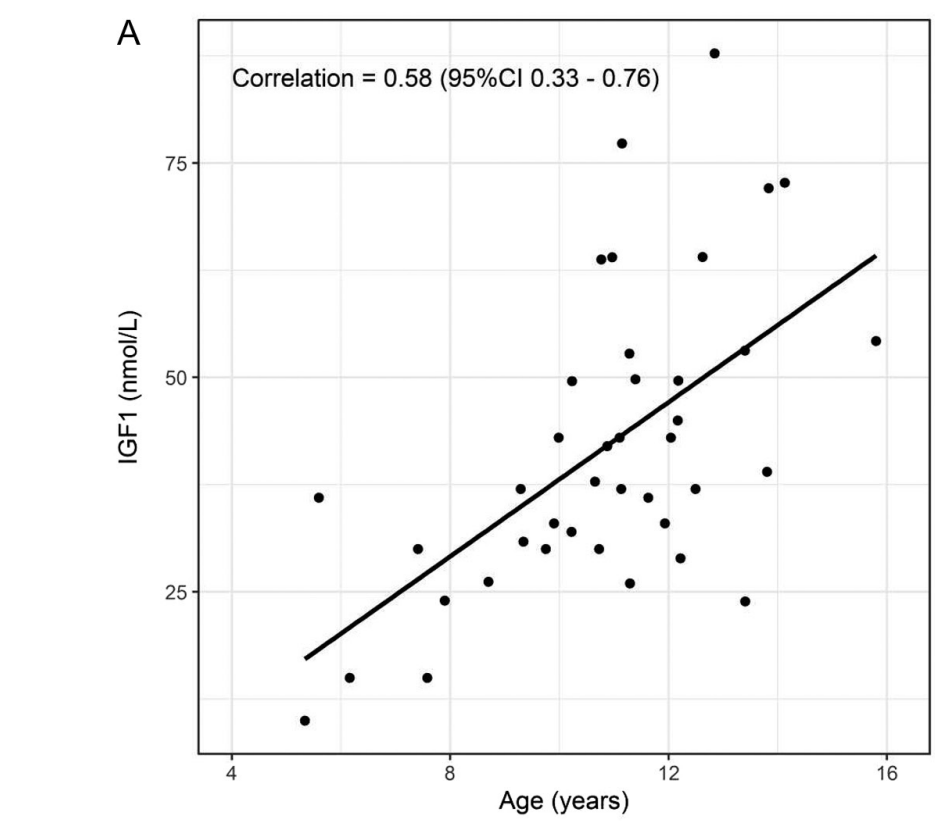

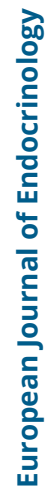

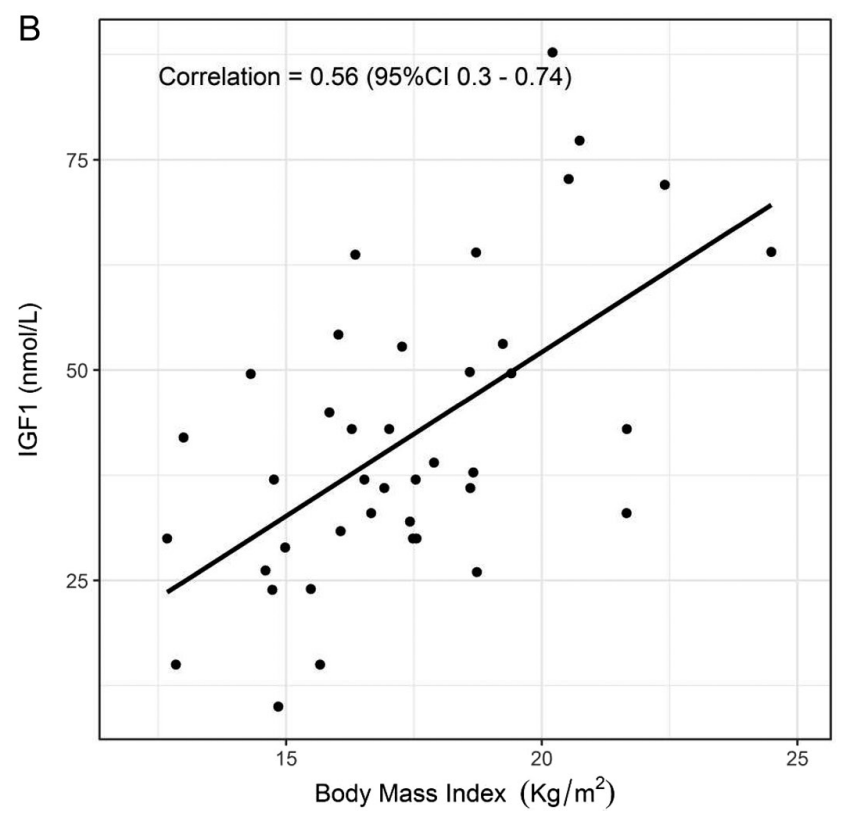

Figure 2

Relationship between (A) IGF-1 and age and between (B) IGF-1 and $\mathrm{BMI}$ after 1 year of treatment.

(stratification by study group is given in Supplementary Table 2). Improvement in auxological data and IGF-1 levels was noted over time for both sexes. Table 3 presents the patients' clinical and auxological data after discontinuation of GH treatment. Statistically significant differences were not detected in definitive height or the delta height between genetic target and definitive height between groups A and B (males: 159.9 vs $159.8 \mathrm{~cm}$,
Table 1 Clinical data of study patients at diagnosis, by gender.

\begin{tabular}{l}
\hline \\
\hline Birth weight (g) \\
Age (years) \\
Prepubertal Stage \\
Bone age (years) \\
$\Delta$ bone age \\
Height (cm) \\
SDS Height \\
Weight $(\mathrm{kg})$ \\
SDS weight \\
BMI (kg/m²) \\
Genetic Target (cm) \\
SDS Genetic Target \\
$\Delta$ SDS height - SDS \\
genetic target \\
GH after arginine ( $\mu \mathrm{gg} / \mathrm{L})$ \\
$\quad(n=80)$ \\
GH after insulin $(\mu \mathrm{g} / \mathrm{L})$ \\
$\quad(n=70)$ \\
GH after glucagon $(\mu \mathrm{Hg} / \mathrm{L})$ \\
$\quad(n=10)$ \\
IGF-1 (nmol/L) \\
SDS IGF-1
\end{tabular}

\begin{tabular}{cccc}
\hline Males $(n=46)$ & & Females $(n=34)$ \\
\cline { 1 - 1 } $2988.11 \pm 612.70$ & & $2994.80 \pm 664.19$ \\
$9.88 \pm 2.69$ & & $9.64 \pm 2.39$ \\
$100 \%$ & & $100 \%$ \\
$8.29 \pm 2.64$ & & $7.94 \pm 2.76$ \\
$-2.16 \pm 1.56$ & & $-1.23 \pm 1.22$ \\
$122.51 \pm 14.89$ & & $122.42 \pm 12.87$ \\
$-2.57 \pm 0.50$ & & $-2.34 \pm 0.38$ \\
$26.90 \pm 8.44$ & & $25.42 \pm 7.54$ \\
$-1.77 \pm 1.04$ & & $-1.82 \pm 0.82$ \\
$17.55 \pm 3.03$ & & $16.58 \pm 2.39$ \\
$169.86 \pm 4.99$ & & $158.17 \pm 4.27$ \\
$-0.97 \pm 0.78$ & & $-0.74 \pm 0.73$ \\
$-1.59 \pm 0.79$ & & $-1.62 \pm 0.82$ \\
$4.60 \pm 2.79$ & & $4.47 \pm 2.27$ \\
$4.85 \pm 2.83$ & & $4.35 \pm 2.33$ \\
$5.55 \pm 1.05$ & & $4.92 \pm 2.99$ \\
$23.13 \pm 12.14$ & $24.75 \pm 10.11$ \\
$-0.84 \pm 1.25$ & & $-0.13 \pm 0.89$ \\
\hline & &
\end{tabular}

$\Delta$, difference; $\mathrm{GH}$, growth hormone; IGF-1, insulin-like growth factor-1; SDS, standard deviation score.

$P=0.637$ and -1.49 vs $-1.51, P=0.826$, respectively, females: 149.2 vs $150.7 \mathrm{~cm}, P=0.944$ and -1.29 vs -1.18 , $P=0.684$, respectively, Table 3 ). Differences in growth velocity between the two groups during the treatment and after discontinuation of therapy were not statistically significant ( 8.0 vs $7.4 \mathrm{~cm} /$ year, $P=0.255$ and 4.4 vs 4.1 $\mathrm{cm} /$ year, $P=0.804)$. Similarly, statistically significant differences were not detected in BA retardation between the two groups at the beginning of treatment or at retesting $(-1.6$ vs $-1.9, P=0.400$ and -1.1 vs $-0.6, P=0.110$, respectively, Supplementary Table 3). A significantly lower growth velocity, expressed as SDS, in the children with persistent GHD was noted during the first year of GH treatment ( 0.043 vs 0.027 SDS, $P=0.046)$. Moreover, in 11 group B patients (30.5\%) the GHD persisted at retesting performed at the end of puberty.

A significant association has been found between patients age and IGF-1 levels at diagnosis (Spearman's rank correlation $\rho=0.46,95 \%$ CI: 0.21 to 0.65 ) and after 1 year of treatment ( $\rho=0.60,95 \%$ CI: 0.36 to 0.76 ; Fig. $2 A$ ). There was a significant relationship between IGF-1 levels and BMI at the time of diagnosis ( $\rho=0.45,95 \%$ CI: 0.20 to 0.65$)$, after 1 year of treatment ( $\rho=0.56,95 \%$ CI: 0.31 to 0.73 , Fig. 2B) and at retesting ( $\rho=0.61,95 \%$ CI: 0.39 to 0.76 ).

We also investigated predictive factors to identify $a$ priori patients with persistent GHD at mid-puberty. We found a nonlinear relationship between IGF-1 levels after 
Table 2 Clinical and auxological data of study patients, stratified by gender, 1 year after the treatment and at puberty.

\begin{tabular}{l}
\hline \\
\hline Age at the beginning of puberty (years) \\
Duration of treatment (years) \\
Data after 1 year of treatment \\
Height (cm) \\
SDS height \\
Weight $(\mathrm{kg})$ \\
BMI (kg/m²) \\
SDS BMI \\
SDS $\Delta$ height \\
Pubertal Stage: P2 \\
Pubertal Stage: T2 \\
Pubertal Stage: testicular volume $4-9 \mathrm{~mL}$ \\
Bone age \\
$\Delta$ bone age \\
IGF-1 (nmol/L) \\
SDS IGF-1 \\
Data at the intermediate stage of puberty \\
Age (years) \\
Height (cm) \\
SDS height \\
Weight (kg) \\
BMI (kg/m²) \\
SDS $\Delta$ height (diagnosis-puberty) \\
IGF-1 (nmol/L) \\
SDS IGF-1
\end{tabular}

\begin{tabular}{c}
\hline Males $(n=46)$ \\
$10.34 \pm 2.49$ \\
$3.97 \pm 2.29$ \\
$132.10 \pm 14.97$ \\
$-2.01 \pm 0.53$ \\
$32.44 \pm 10.15$ \\
$17.63 \pm 2.90$ \\
$-0.66 \pm 1.18$ \\
$0.54 \pm 0.39$ \\
$6 / 46(13.0 \%)$ \\
$/$ \\
$9 / 46(19.6 \%)$ \\
$9.44 \pm 2.75$ \\
$-1.80 \pm 1.48$ \\
$39.42 \pm 18.42$ \\
$0.31 \pm 0.96$ \\
$12.23 \pm 1.28$ \\
$137.98 \pm 7.02$ \\
$-1.92 \pm 0.57$ \\
$35.26 \pm 6.34$ \\
$18.67 \pm 2.76$ \\
$0.63 \pm 0.54$ \\
$56.54 \pm 17.21$ \\
$0.21 \pm 0.84$ \\
\end{tabular}

\begin{tabular}{c}
\hline Females $(n=34)$ \\
\hline $9.83 \pm 2.44$ \\
$3.01 \pm 1.64$ \\
$131.29 \pm 13.33$ \\
$-1.87 \pm 0.43$ \\
$29.81 \pm 9.05$ \\
$16.88 \pm 2.58$ \\
$-0.78 \pm 1.04$ \\
$0.46 \pm 0.42$ \\
$6 / 34(17.6 \%)$ \\
$7 / 34(20.6 \%)$ \\
$/$ \\
$9.65 \pm 2.95$ \\
$0.97 \pm 0.60$ \\
$46.93 \pm 14.97$ \\
$0.65 \pm 0.83$ \\
$11.44 \pm 1.39$ \\
$135.26 \pm 5.88$ \\
$-1.84 \pm 0.54$ \\
$31.41 \pm 5.91$ \\
$17.10 \pm 2.59$ \\
$0.51 \pm 0.59$ \\
$60.53 \pm 18.61$ \\
$0.99 \pm 0.84$ \\
\end{tabular}

$\Delta$, difference; IGF-1, insulin-like growth factor-1; SDS, standard deviation score.

Table 3 Comparison of clinical and auxological data between patients of group A (normal GH secretion) vs group B (persistent GHD), stratified by gender, after the suspension of GH treatment.

\begin{tabular}{l} 
\\
\hline Data at retesting \\
Age (years) \\
Height (cm) \\
SDS height \\
Weight (kg) \\
SDS weight \\
BMI (kg/m²) \\
SDS $\Delta$ height (restesting - diagnosis) \\
SDS $\Delta$ height (retesting - puberty) \\
SDS $\Delta$ height (retesting - after 1year \\
of treatment) \\
Bone age \\
$\Delta$ bone age \\
GH after arginine ( $\mu$ g/L) \\
IGF-1 (nmol/L) \\
SDS IGF-1 \\
Genetic Target (cm) \\
SDS Genetic Target \\
Near adult data \\
Near adult height (cm) \\
SDS near adult height \\
$\Delta$ Height - Genetic target (cm) \\
$\Delta$ SDS Height - SDS genetic target \\
\hline
\end{tabular}

\begin{tabular}{c} 
Group A (normal G \\
\hline Males $(n=23)$ \\
\hline $14.36 \pm 1.50$ \\
$154.40 \pm 9.73$ \\
$-1.39 \pm 0.36$ \\
$45.19 \pm 8.71^{*}$ \\
$-1.33 \pm 1.01^{*}$ \\
$18.82 \pm 2.52^{* *}$ \\
$1.16 \pm 0.51$ \\
$0.53 \pm 0.41$ \\
$0.57 \pm 0.37$ \\
$13.62 \pm 0.99$ \\
$-1.23 \pm 0.94$ \\
$17.10 \pm 8.33$ \\
$58.12 \pm 7.55$ \\
$0.05 \pm 0.43$ \\
$170.70 \pm 3.22$ \\
$-0.84 \pm 0.51$ \\
$159.90 \pm 5.64$ \\
$-1.49 \pm 0.69$ \\
$-10.79 \pm 5.53$ \\
$-0.69 \pm 0.69$ \\
\hline
\end{tabular}

GH secretion; $n=44)$
Females $(n=21)$

$13.58 \pm 1.57$

$148.16 \pm 8.12$

$-1.21 \pm 0.79$

$41.48 \pm 9.25$

$-1.44 \pm 0.75$

$18.73 \pm 3.28$

$1.08 \pm 0.85$

$0.76 \pm 0.86$

$0.47 \pm 0.52$

$12.11 \pm 1.81$

$-0.82 \pm 0.73$

$16.69 \pm 9.71$

$55.83 \pm 11.77$

$0.18 \pm 0.81$

$158.64 \pm 4.30$

$-0.66 \pm 0.74$

$149.19 \pm 8.93$

$-1.29 \pm 0.55$

$-10.73 \pm 8.37$

$-0.70 \pm 0.73$

\begin{tabular}{|c|c|}
\hline \multicolumn{2}{|c|}{ Group B (persistent GHD; $n=36$ ) } \\
\hline Males $(n=23)$ & Females $(n=13)$ \\
\hline $14.39 \pm 1.21$ & $12.65 \pm 1.19$ \\
\hline $153.93 \pm 8.08$ & $144.57 \pm 5.68$ \\
\hline$-1.47 \pm 0.61$ & $-1.38 \pm 0.67$ \\
\hline $51.60 \pm 11.15$ & $37.68 \pm 7.29$ \\
\hline$-0.67 \pm 1.06$ & $-1.43 \pm 1.05$ \\
\hline $21.68 \pm 4.30$ & $17.94 \pm 2.67$ \\
\hline $1.21 \pm 0.56$ & $1.03 \pm 0.68$ \\
\hline $0.47 \pm 0.44$ & $0.32 \pm 0.36$ \\
\hline $0.62 \pm 0.49$ & $0.69 \pm 0.46$ \\
\hline $13.94 \pm 0.82$ & $12.51 \pm 1.27$ \\
\hline$-0.66 \pm 0.95$ & $-0.53 \pm 0.94$ \\
\hline $3.85 \pm 2.15$ & $4.04 \pm 2.52$ \\
\hline $55.25 \pm 17.16$ & $47.00 \pm 22.88$ \\
\hline$-0.22 \pm 0.97$ & $-0.09 \pm 1.23$ \\
\hline $168.91 \pm 6.38$ & $157.45 \pm 4.31$ \\
\hline$-1.12 \pm 1.00$ & $-0.87 \pm 0.73$ \\
\hline $159.76 \pm 7.56$ & $150.66 \pm 5.06$ \\
\hline$-1.51 \pm 0.66$ & $-1.18 \pm 0.60$ \\
\hline$-8.43 \pm 9.81$ & $-6.56 \pm 6.19$ \\
\hline$-0.34 \pm 1.14$ & $-0.28 \pm 0.83$ \\
\hline
\end{tabular}

${ }^{*} P<0.05$ males group A vs males group $B ; * * P<0.01$ males group A vs males group $B$.

$\Delta$, difference; GH, growth hormone; IGF-1, insulin-like growth factor-1; SDS, standard deviation score. 
1 year of treatment and the risk of GHD (Figure 1). A logistic regression model with a linear spline on the covariate IGF-1 was used to approximate this relationship; for IGF-1 levels below the breakpoint of $50 \mathrm{nmol} / \mathrm{L}$, the risk of GHD significantly increased with decreasing IGF-1 levels (odds ratio $(\mathrm{OR})=0.91,95 \% \mathrm{CI}: 0.84$ to $0.98 ; P=0.019)$. The model has a good predictive power (AUC $=0.75,95 \%$ CI: 0.60 to 0.90 ). In detail, an IGF-1 level of $<32.5 \mathrm{nmol} / \mathrm{L}$ after 1 year of treatment is indicative of persistent GHD (67\% sensitivity, 76\% specificity). When we performed the same analysis for IGF-1 SDS after 1 year of treatment, again we found a nonlinear relationship. For IGF-1 below the breakpoint of 0 SDS, the risk of GHD significantly increases with decreasing IGF-1 levels (OR=0.13, 95\% CI: 0.02 to $0.97 ; P=0.047)$. The model demonstrated good predictive power (AUC $=0.78,95 \% \mathrm{CI}$ : 0.64 to 0.92 ) and IGF-1 SDS levels <-0.45 seem indicative of persistent GHD (84\% sensitivity, 63\% specificity). In the subgroup of patients with IGF-1 SDS $<0$, the sensitivity and the specificity associated with the threshold of -0.45 were 80\% (95\% CI: 28.4 to $99.5 \%$ ) and $88.9 \%$ (95\% CI: $51.8 \%$ to $99.7 \%)$, respectively.

We did not detect significant relationships between GHD at mid-puberty and IGF-1 levels at diagnosis and at retesting (22.7 vs $24.9 \mathrm{nmol} / \mathrm{L}, P=0.499$ and 57.0 vs 52.3 $\mathrm{nmol} / \mathrm{L}, \quad P=0.313$, respectively; Supplementary Tables 1 and 3). No other predictive factors were identified. At retesting, however, we found high IGF-1 levels (and IGF-1 SDS) and a significant linear relationship between these values and the BMI in the patients with persistent GHD (slope $=1.82,95 \%$ CI: $0.55-3.10, P=0.006$, and slope $=0.15$, 95\% CI: $0.05-0.25, P=0.004$, respectively).

\section{Discussion}

Here we report on the advantage of reexamining at puberty children with idiopathic GHD treated with GH for at least 2 years during childhood. In more than $50 \%$ of patients retested during the intermediate stage of puberty, GH treatment was discontinued at this point, avoiding unnecessary years of treatment. When we anticipate retesting to mid-puberty, we found no evidence for a reduction in final height, both in terms of adult height and in relation to their genetic target. These data are in line with a previous study in which only one-third of patients discontinued treatment at mid-puberty (6). Our findings contrast with other studies that, on the contrary, suggested increasing the GH dose during puberty in order to optimize the GH effect $(18,19)$.
GH secretion normally rises two- to four-fold during the pubertal growth spurt, and maximum GH secretion coincides with pubertal peak (20). Nevertheless, our results hold interest for their implication for treatment costs and benefits. GH therapy is very expensive. It has been estimated that the annual treatment for a $30-\mathrm{kg}$ child costs EUR 4.650 (93.6\% drug cost and 6.4\% monitoring cost), that the incremental cost of GH treatment for one child with GHD (for 5-8 years of GH treatment) ranges from EUR 33.900 to EUR 40.700 and that each centimeter increment in final height gained due to GH treatment costs approximately EUR 4.400-4.600 (21). Moreover, as GHD patients become taller and heavier as they advance in age during treatment, the cost for GH treatment rises. This economic analysis is in line with the costs that the Italian Health System estimates for GH treatment and with NICE guidelines, which suggest that GH costs approximately EUR 7.355 per $\mathrm{cm}$ final height on average for idiopathic GHD (3). A previous study showed that higher absolute doses of GH produce little benefit in height gain after pubertal onset but rather simply raise the treatment costs (22). In this context, our results might offer a good alternative: it might be beneficial to retest all GHD patients at mid-puberty and then evaluate in a subsequent study whether only patients with persistent GHD benefit from higher doses in order to improve their final stature. This clinical strategy might significantly reduce treatment costs. The same authors who suggested that high dose GH therapy may have beneficial effects on pubertal patients with GHD appeared skeptical, however, about this approach because of the high cost of GH treatment (23). Furthermore, retesting GHD in midpuberty can satisfy patients' expectations of treatment effect and assuage concerns over potential side effects. Despite data on the long-term safety of GH treatment (24, 25 ) and the absence of adverse effects in both groups of our patients, a reduction in the time of exposure to $\mathrm{GH}$ treatment may also reduce the possibility of later side effects.

The only factor with a significant ability to identify GHD patients at mid-puberty retest seems to be the IGF-1 level and IGF-1 SDS after 1 year of treatment. On the contrary, IGF-1 levels and IGF-1 SDS before and after GH treatment did not result as a predictive factor for normal $\mathrm{GH}$ secretion and we found no any other factor (growth velocity, BMI, delta height SDS) that could predict GH sufficiency at puberty. Our study findings suggest that, if IGF-1 levels after 1 year of treatment remain $<32.5 \mathrm{nmol} / \mathrm{L}$ (or IGF- $1<-0.45$ SDS) despite GH therapy, children might not be retested at mid-puberty. These data result from 
logistic regression model with a good predictive power, but these cutoff values need to be confirmed in larger patients' population through appropriate prospective clinical trials. In agreement with some previous investigations (26) but not with others (27), our data showed that the response to therapy after the first year of treatment differs between children with persistent deficiency and those with normalized secretion. Moreover, our results agree with previous studies $(6,27,28)$ that did not detect significant correlation between IGF-1 levels and the GH peak at retesting. However, IGF-1 levels have a good sensitivity only in detecting subjects with severe GHD, while the overlap between normal and GHD subjects is significant when a GH cutoff like ours is used $(6,29,30)$. This overlap is more accentuated by the fact that patients are taking or have just finished GH therapy. In line with this observation, other authors found IGF-1 levels of around 0 s.D. in adolescents with partial GHD at the end of treatment (31).

Our findings show that GH levels at diagnosis and treatment duration were not predictive for future growth. Therefore, the statural gain after diagnosis does not seem to depend on GH response to pharmacological stimulation. In regard to the issue of the utility of stimulus testing for GH diagnosis $(32,33)$, our data confirm that a consistent percentage of children diagnosed with GHD showed no deficiency when reexamined at mid-puberty. The biochemical diagnosis of GHD traditionally requires the demonstration of absent or low GH levels in response to GH-stimulation protocols that test the GH-IGF-1 axis in a non-physiological manner with agents of different strength of stimulus to axis and that act at various different levels but have poor reproducibility (34). The commonly used peak GH cutoff value has not been adjusted for factors (e.g., age, sex, pubertal status and adiposity) that affect GH secretion throughout childhood (33). This factor may lead to misdiagnosis of GHD. Studies suggest that, depending on the series and the cutoff used for diagnosis, 60 to $85 \%$ of patients diagnosed with GHD in childhood will have adequate GH secretion when retested in late adolescence or adulthood, which may, at least in part, represent maturation of the pituitary gland $(6,14,26,35$, 36 ). We are aware that the reliability of pharmacological testing is largely controversial, particularly in subjects with non-severe GHD $(4,5,37,38)$, and that the GH cutoff limits have been arbitrarily established without taking into account the physiological rise in GH secretion during puberty (6). Moreover, it is known that there are different assay methods for GH evaluation suggesting assay-specific interpretation of provocation test of
GH disorders (39). Therefore, based on the previously mentioned considerations, the clinical and biochemical diagnosis of GH continue to be a conundrum. It is of utmost importance to select children correctly to be tested and to establish the correct timing of retesting in order to avoid potential side effects and added pharmaceutical costs.

Our study has several limitations: the retrospective review design, the small sample size and the lack of information on drug compliance. While only rigorously monitored and controlled trials can exclude the influence of non-compliance, the present study adequately reflects the reality of daily clinical care. Anyhow, our patients reported a good compliance to GH treatment, optimal in the first year of therapy and progressively lower with increasing duration of therapy. Finally, we did not consider IGFBP3 in GHD and follow-up. IGFBP3 measurement may be helpful in monitoring adherence to and safety of GH treatment, but it seems insufficient to confirm GHD and it is not useful to adjust the GH dose needed to treat prepubertal children with GHD $(40,41)$.

In conclusion, our study suggests that, in children with idiopathic and isolated GHD, GH secretion should be retested at mid-puberty. Discontinuation of treatment in children who no longer show GHD does not seem to impair growth potential and adult height. On the contrary, in such cases, discontinuation may reduce potential side effects and unnecessary discomfort to patients and their families, as well as reduce costs for the National Health care system. A future area of investigation is to determine whether pubertal patients with persistent GHD can benefit from higher GH doses to further improve their final stature.

\section{Supplementary materials}

This is linked to the online version of the paper at https://doi.org/10.1530/ EJE-19-0646.

Declaration of interest

The authors declare that there is no conflict of interest that could be perceived as prejudicing the impartiality of this study.

\section{Funding}

This research did not receive any specific grant from any funding agency in the public, commercial or not-for-profit sector.

\section{References}

1 Antoniazzi F, Cavarzere P \& Gaudino R. Growth hormone and early treatment. Minerva Endocrinologica 201540 129-143. 
2 Murray PG, Dattani MT \& Clayton PE. Controversies in the diagnosis and management of growth hormone deficiency in childhood and adolescence. Archives of Disease in Childhood 2016101 96-100. (https://doi.org/10.1136/archdischild-2014-307228)

3 National Institute for Health and Care Excellence. TA188. Human growth hormone (somatropin) for the treatment of growth failure in children: guidance, 2013. (available at: https://www.nice.org.uk/ guidance/ta188)

4 Growth Hormone Research Society. Consensus guidelines for the diagnosis and treatment of growth hormone (GH) deficiency in childhood and adolescence: summary statement of the GH Research Society. GH Research Society. Journal of Clinical Endocrinology and Metabolism 200085 3990-3993. (https://doi.org/10.1210/ jcem.85.11.6984)

5 Maghnie M, Strigazzi C, Tinelli C, Autelli M, Cisternino M, Loche S \& Severi F. Growth hormone (GH) deficiency (GHD) of childhood onset: reassessment of GH status and evaluation of the predictive criteria for permanent GHD in young adults. Journal of Clinical Endocrinology and Metabolism 199984 1324-1328. (https://doi. org/10.1210/jcem.84.4.5614)

6 Zucchini S, Pirazzoli P, Baronio F, Gennari M, Orquidea Bal MO, Balsamo A, Gualandi S \& Cicognani A. Effect on adult height of pubertal growth hormone retesting and withdrawal of therapy in patients with previously diagnosed growth hormone deficiency. Journal of Clinical Endocrinology and Metabolism 200691 4271-4276. (https://doi.org/10.1210/jc.2006-0383)

7 Reiter EO, Price DA, Wilton P, Albertsson-Wikland K \& Ranke MB. Effect of growth hormone (GH) treatment on the near-final height of 1258 patients with idiopathic GH deficiency: analysis of a large international database. Journal of Clinical Endocrinology and Metabolism 200691 2047-2054. (https://doi.org/10.1210/jc.20052284)

8 Root AW, Dana K \& Lippe B. Treatment of growth hormone-deficient infants with recombinant human growth hormone to near-adult height: patterns of growth. Hormone Research in Paediatrics 201175 276-283. (https://doi.org/10.1159/000322881)

9 Martha Jr PM, Rogol AD, Carlsson LM, Gesundheit N \& Blizzard RM. A longitudinal assessment of hormonal and physical alterations during normal puberty in boys. I. Serum growth hormone-binding protein. Journal of Clinical Endocrinology and Metabolism $1993 \mathbf{7 7}$ 452-457. (https://doi.org/10.1210/jcem.77.2.8345051)

10 Ranke MB \& Lindberg A. Observed and predicted total pubertal growth during treatment with growth hormone in adolescents with idiopathic growth hormone deficiency, Turner syndrome, short stature, born small for gestational age and idiopathic short stature: KIGS analysis and review. Hormone Research in Paediatrics 201175 423-432. (https://doi.org/10.1159/000324117)

11 Ranke MB, Lindberg A, Brosz M, Kaspers S, Loftus J, Wollmann H, Kołtowska-Haggstrom M \& Roelants M. Accurate long-term prediction of height during the first four years of growth hormone treatmentin prepubertal children with growth hormone deficiency or Turner syndrome. Hormone Research in Paediatrics 201278 8-17. (https://doi.org/10.1159/000339468)

12 Smuel K, Kauli R, Lilos P \& Laron Z. Growth, development, puberty and adult height before and during treatment in children with congenital isolated growth hormone deficiency. Growth Hormone and IGF Research 201525 182-188. (https://doi.org/10.1016/j. ghir.2015.05.001)

13 Rose SR, Municchi G, Barnes KM, Kamp GA, Uriarte MM, Ross JL, Cassorla F \& Cutler Jr GB. Spontaneous growth hormone secretion increases during puberty in normal girls and boys. Journal of Clinical Endocrinology and Metabolism 199173 428-435. (https://doi. org/10.1210/jcem-73-2-428)

14 Bizzarri C, Pedicelli S, Boscherini B, Bedogni G, Cappa M \& Cianfarani S. Early retesting for growth hormone (GH) secretion avoids useless extensions of GH treatments in children with idiophatic isolated GH deficiency. Journal of Endocrinological Investigation 201538 429-436. (https://doi.org/10.1007/s40618-0140205-3)

15 Richmond E \& Rogol AD. Treatment of growth hormone deficiency in children, adolescents and at the transitional age. Best Practice and Research: Clinical Endocrinology and Metabolism 201630 749-755. (https://doi.org/10.1016/j.beem.2016.11.005)

16 Tanner JM \& Whitehouse RH. Clinical longitudinal standards for height, weight, height velocity, weight velocity, and stages of puberty. Archives of Disease in Childhood $1976 \mathbf{5 1}$ 170-179. (https:// doi.org/10.1136/adc.51.3.170)

17 Greulich WW \& Pyle SI. Radiographic Atlas of Skeletal Development of the Hand and Wrist, 2nd ed. Stanford: Stanford University Press, 1959.

18 Albertsson-Wikland K, Kriström B, Lundberg E, Aronson AS, Gustafsson J, Hagenäs L, Ivarsson SA, Jonsson B, Ritzén M, Tuvemo T et al. Growth hormone dose-dependent pubertal growth: a randomized trial in short children with low growth hormone secretion. Hormone Research in Paediatrics 201482 158-170. (https:// doi.org/10.1159/000363106)

19 Mauras N, Attie KM, Reiter EO, Saenger P \& Baptista J. High dose recombinant human growth hormone (GH) treatment of GH-deficient patients in puberty increases near-final height: a randomized, multicenter trial. Genentech, Inc., Cooperative Study Group. Journal of Clinical Endocrinology and Metabolism 200085 3653-3660. (https://doi.org/10.1210/jcem.85.10.6906)

20 Martha PM, Rogol AD, Veldhius JD, Keerigan JR, Godman DW \& Blizzard RM. Alterations in the pulsatile properties of circulating growth hormone concentrations during puberty in boys. Journal of Clinical Endocrinology and Metabolism 198969 563-570. (https://doi. org/10.1210/jcem-69-3-563)

21 Takeda A, Cooper K, Bird A, Baxter L, Frampton GK, Gospodarevskaya E, Welch K \& Bryant J. Recombinant human growth hormone for the treatment of growth disorders in children: a systematic review and economic evaluation. Health Technology Assessment 201014 1-209. (https://doi.org/10.3310/hta14420)

22 Howard SR \& Butler GE. An analysis of the clinical and cost effectiveness of growth hormone replacement therapy before and during puberty: should we increase the dose? Hormone Research in Paediatrics 201379 75-82. (https://doi.org/10.1159/000346687)

23 Riddick L, Alter C, Davis DA, Frane J, Lippe B \& Bakker B. A stepwise increase in recombinant human growth hormone dosing during puberty achieves improved pubertal growth: a National Cooperative Growth Study report. Journal of Pediatric Endocrinology and Metabolism 200922 623-628. (https://doi.org/10.1515/jpem.2009.22.7.623)

24 Stochholm K \& Kiess W. Long-term safety of growth hormone - a combined registry analysis. Clinical Endocrinology 201888 515-528. (https://doi.org/10.1111/cen.13502)

25 Carel JC \& Butler G. Safety of recombinant human growth hormone. Endocrine Development 201018 40-54. (https://doi. org $/ 10.1159 / 000316126)$

26 Thomas M, Massa G, Maes M, Beckers D, Craen M, Francois I, Heinrichs C, Bourguignon JP \& Belgian Study Group for Paediatric Endocrinology (BSGPE). Growth hormone (GH) secretion in patients with childhood onset GH deficiency: retesting after one year of therapy and at final height. Hormone Research 200359 7-15. (https:// doi.org/10.1159/000067936)

27 Loche S, Bizzarri C, Maghnie M, Faedda A, Tzialla C, Autelli M, Casini MR \& Cappa M. Results of early reevaluation of growth hormone secretion in short children with apparent growth hormone deficiency. Journal of Pediatrics 2002140 445-449. (https://doi. org/10.1067/mpd.2002.122729)

28 Hoeck HC, Vestergaard P, Jakobsen PE, Falhof J \& Laurberg P. Diagnosis of growth hormone (GH) deficiency in adults with hypothalamic-pituitary disorders: comparison of test results using pyridostigmine plus GH-releasing hormone (GHRH), clonidine plus GHRH, and insulin-induced hypoglycemia as GH secretagogues. 
Journal of Clinical Endocrinology and Metabolism 200085 1467-1472. (https://doi.org/10.1210/jcem.85.4.6538)

29 Darendeliler F, Spinu I, Bas F, Bundak R, Isguven P, Arslanoglu I, Saka N, Sukur M \& Gunoz H. Reevaluation of growth hormone deficiency during and after growth hormone $(\mathrm{GH})$ treatment: diagnostic value of GH tests and IGF-I and IGFBP-3 measurements. Journal of Pediatric Endocrinology and Metabolism 200417 1007-1012. (https://doi.org/10.1515/jpem.2004.17.7.1007)

30 Juul A \& Skakkebaek NE. Prediction of the outcome of growth hormone provocative testing in short children by measurement of serum levels of insulin-like growth factor I and insulin-like growth factor binding protein 3. Journal of Pediatrics 1997130 197-204. (https://doi.org/10.1016/s0022-3476(97)70343-3)

31 Tauber M, Jouret B, Cartault A, Lounis N, Gayrard M, Marcouyeux C, Pienkowski C, Oliver I, Moulin P, Otal P et al. Adolescents with partial growth hormone (GH) deficiency develop alterations of body composition after GH discontinuation and require follow-up. Journal of Clinical Endocrinology and Metabolism 200388 5101-5106. (https:// doi.org/10.1210/jc.2003-030392)

32 Alatzoglou KS, Webb EA, Le Tissier P \& Dattani MT. Isolated growth hormone deficiency (GHD) in childhood and adolescence: recent advances. Endocrine Reviews 201435 376-432. (https://doi. org/10.1210/er.2013-1067)

33 Stanley T. Diagnosis of growth hormone deficiency in childhood. Current Opinion in Endocrinology, Diabetes, and Obesity 201219 47-52. (https://doi.org/10.1097/MED.0b013e32834ec952)

34 Richmond EJ \& Rogol AD. Growth hormone deficiency in children. Pituitary 200811 115-120. (https://doi.org/10.1007/s11102-008-0105-7)

35 Tauber M, Moulin P, Pienkowski C, Jouret B \& Rochiccioli P. Growth hormone $(\mathrm{GH})$ retesting and auxological data in 131GH-deficient patients after completion of treatment. Journal of Clinical Endocrinology and Metabolism 199782 352-356. (https://doi. org/10.1210/jcem.82.2.3726)

36 Meazza C, Gertosio C, Pagani S, Pilotta A, Tinelli C, Buzi F, Farello G, Genoni $\mathrm{G}$, Bona $\mathrm{G} \&$ Bozzola $\mathrm{M}$. Is retesting in growth hormone deficient children really useful? Minerva Endocrinologica 201742 325-330. (https://doi.org/10.23736/S0391-1977.16.02510-4)

37 Ghigo E, Bellone J, Aimaretti G, Bellone S, Loche S, Cappa M, Bartolotta E, Dammacco F \& Camanni F. Reliability of provocative tests to assess growth hormone secretory status. Study in 472 normally growing children. Journal of Clinical Endocrinology and Metabolism 199681 3323-3327. (https://doi.org/10.1210/ jcem.81.9.8784091)

38 Gandrud LM \& Wilson DM. Is growth hormone stimulation testing in children still appropriate? Growth Hormone and IGF Research 2004 14 185-194. (https://doi.org/10.1016/j.ghir.2003.11.003)

39 Van Helden J, Hermsen D, Von Ahsen N \& Bidlingmaier M. Performance evaluation of a fully automated immunoassay for the detection of human growth hormone on the Elecsys immunoassay system. Clinical Laboratory 201460 1641-1651. (https://doi. org/10.7754/clin.lab.2014.131020)

40 Lanes R \& Jakubowicz S. Is insulin-like growth factor-1 monitoring useful in assessing the response to growth hormone of growth hormone-deficient children? Journal of Pediatrics 2002141 606-610. (https://doi.org/10.1067/mpd.2002.127662)

41 Ali A, Hashim R, Khan FA, Sattar A, Ijaz A, Manzoor SM \& Younas M. Evaluation of insulin-like growth factor-1 and insulin like growth factor binding protein-3 in diagnosis of growth hormone deficiency in short-stature children. Journal of Ayub Medical College, Abbottabad 200921 40-45.

Received 16 August 2019

Revised version received 24 March 2020

Accepted 27 March 2020 